\title{
Infrared ship signature prediction, model validation and sky radiance
}

\author{
Filip Neele* \\ TNO Defence, Security and Safety, The Hague, The Netherlands
}

\begin{abstract}
The increased interest during the last decade in the infrared signature of (new) ships results in a clear need of validated infrared signature prediction codes. This paper presents the results of comparing an in-house developed signature prediction code with measurements made in the 3-5 $\mu \mathrm{m}$ band in both clear-sky and overcast conditions. During the measurements, sensors measured the short-wave and long-wave irradiation from sun and sky, which forms a significant part of the heat flux exchange between ship and environment, but is linked weakly to the standard meteorological data measured routinely (e.g., air temperature, relative humidity, wind speed, pressure, cloud cover). The aim of the signature model validation is checking the heat flux balance algorithm in the model and the representation of the target. Any uncertainties in the prediction of the radiative properties of the environment (which are usually computed with a code like MODTRAN) must be minimised. It is shown that for the validation of signature prediction models the standard meteorological data are insufficient for the computation of sky radiance and solar irradiation with atmospheric radiation models (MODTRAN). Comparisons between model predictions and data are shown for predictions computed with and without global irradiation data. The results underline the necessity of measuring the irradiation (from sun, sky, sea or land environment) on the target during a signature measurement trial. Only then does the trial produce the data needed as a reference for the computation of the infrared signature of the ship in conditions other than those during the trial.
\end{abstract}

Keywords: Ships, infrared signature, signature prediction model, model validation

\section{INTRODUCTION}

Recent years show an increasing awareness of the impact of a ship's susceptibility to the IR guided threat on its mission effectiveness. A number of low-observable ships are currently being designed and built. As a result, there is a clear need for validated infrared signature prediction models and recently several papers have been addressed to the validation method and to the validation of ship infrared signature prediction models ${ }^{1-3}$. As the infrared signature strongly depends on the environment and the ship's configuration, a complete description of the infrared signature of a ship can only be obtained through modelling of the signature in a range of operational environments ${ }^{4}$. The signature prediction model must be validated with measurements, preferably in a (wide) range of atmospheric conditions that covers the atmospheric conditions expected in the ship's operational areas.

This paper discusses the validation of infrared signature prediction models. As noted above, the atmospheric environment plays a central role in the generation of the infrared signature. Essentially, the computation of an infrared signature is a two-stage process. First, for a given set of meteorological data, the radiance of sun, sky and sea must be computed. In the second stage, these results, combined with internal heat sources and physical properties and orientation of the ship, serve as input to a heat flux balance, which yields the ship's equilibrium temperature and radiance. Signature prediction models typically use external models for the computation of the radiance of sun, sky and sea. Therefore, the validation of an infrared signature prediction code should deal with the second step only and the influence of errors in the representation of the trial environment (sun and sky radiance) in the signature modelling should be minimised. This paper discusses the use of global irradiation sensors (pyranometer and pyrgeometer) to this end. With data from a recent signature measurement trial, it is demonstrated that the data from these sensors are essential in matching the predicted atmospheric environment to the trial environment.

\footnotetext{
* filip.neele@ tno.nl; phone +31 70374 0461; fax +31 703740654
} 


\section{SIGNATURE MEASUREMENTS}

In June 2003, near the North Sea coast at Noordwijk, The Netherlands, infrared signature measurements were conducted on one the ships of the Royal Netherlands Navy. The trial location was at $52.23{ }^{\circ} \mathrm{N}, 4.42{ }^{\circ} \mathrm{E}$. During the trial meteorological data were collected to support signature modelling that was done after the trial. The data are listed in Table 1. During the trial, a total of five runs were performed, at various times of the day and under different atmospheric conditions. The data in the table represent average values over a period of approximately 30 minutes prior to the actual measurements, during which time the ship maintained course and speed to ensure thermal equilibrium. The ship was required to be in thermal equilibrium for a valid comparison between model prediction and observations, as the signature prediction model used only computes results for thermal equilibrium (see below).

The meteorological data listed in the table were collected both at the measurement site on the shore and on the ship. The data from the ship were used to define air temperature and relative humidity, as the data from the shore-based instruments are not representative for the optical path over the sea. Wind direction and speed was taken from the shorebased instruments. The standard equipment on the shore was extended with a pyranometer (short-wave irradiation) and a pyrgeometer (long-wave irradiation). These sensors were oriented horizontally (pointing upwards), to measure the total irradiation from sun and sky. The sensors operate in the wavelength bands $0.33-2.2 \mu \mathrm{m}$ (pyranometer) and $4.2-40 \mu \mathrm{m}$ (pyrgeometer).

In addition to the shore-based and ship-based meteorological data, temperature sensors were placed in various locations on the ship's surface. These were used as a reference for correction of atmospheric effects of the infrared images and also provided the data to check thermal equilibrium of the ship during the measurement.

In the sections below, physical and apparent temperatures from the port and starboard sides of the bridge section of the ship are used in the comparison between model and observations. The physical temperatures were taken from the temperature sensors on the ship, while the apparent temperature was derived from midwave (3-5 $\mu$ m) images. During most runs these images were taken of the starboard side of the ship; during run IP8 the port side was facing the camera. Average temperatures were determined for the bridge section of the topside, in an area approximately $10 \times 10 \mathrm{~m}^{2}$. The normal of this section of ship is oriented perpendicular to the ship's heading, with a slight elevation. During runs IP1 and IP6, the starboard side of the ship was illuminated (and heated) by the sun. No long-wave infrared data are used in this report, due to the lower spatial resolution of the long-wave infrared imager used during the trial.

\section{METHOD}

\subsection{Signature prediction code}

The infrared signatures shown in the sections below, in the form of apparent and physical target temperatures, were computed with the model EOSM, developed at TNO. This code takes into account the relevant heat fluxes in the maritime environment: irradiance from sea, sun and sky in the entire electro-optical domain (UV to long-wave infrared), convective fluxes with the ambient air and heat conduction to or from the interior. All calculations are fully spectrally resolved and the directionality of irradiation from the sun and sky is accounted for. The input to the code consists of solar irradiation, directional sky radiance (in tabulated form, computed with MODTRAN ${ }^{5}$ ), emissivity of the ship's coating system, orientation of the ship's facets, type and thickness of insulation and type and thickness of the construction material. The code has been validated using both scale model measurements and data from ranging trials on ships. The EOSM code is part of the EOSTAR model $^{6}$.

\subsection{Solar irradiance and sky radiance}

The data in Table 1 were used as input to the atmospheric transmission and radiance code MODTRAN 4.0, to compute solar irradiation and sky radiance. As sky radiance depends on azimuth and elevation, the sky hemisphere was divided into (approximately) equal-area sections about $15^{\circ}$ wide, resulting in a total of 104 combinations of azimuth and elevation. The number of azimuthal steps increases with increasing zenith angle. 
Two atmospheric data sets were computed, to be used as input for the EOSM model. The first set is constructed by using the standard meteorological data (air temperature, relative humidity, pressure) and taking cloud cover into account only through selection of the MODTRAN standard cloud model 4 (stratus/stratocumulus). The geographical location, day of the year and time of day were also input to define the position of the sun. In this first set, no attempt was made to match MODTRAN sky radiance or solar irradiation to the observed values.

Table 1: Meteorological parameters for five signature measurement runs analysed in this paper.

\begin{tabular}{|c|c|c|c|c|c|}
\hline Run & IP1 & IP4 & IP6 & IP7 & IP8 \\
\hline Local time & June $17,11: 40$ & June $17,15: 35$ & June $18,13: 20$ & June $18,17: 40$ & June $18,18: 35$ \\
\hline Pressure (mbar) & 1010 & 1008 & 1017 & 1018 & 1017 \\
\hline Air temperature $\left({ }^{\circ} \mathrm{C}\right)$ & 20.9 & 22.1 & 15.3 & 17.0 & 16.0 \\
\hline Relative humidity (\%) & 53 & 53 & 89 & 71 & 76 \\
\hline Effective wind speed $(\mathrm{m} / \mathrm{s})$ & 8.5 & 9.5 & 5.8 & 4.4 & 25 \\
\hline Wind direction $\left({ }^{\circ}, \mathrm{E}\right.$ of $\left.\left.\mathrm{N}\right)\right)$ & 85 & 63 & 278 & 274 & 52 \\
\hline Heading $\left({ }^{\circ}, \mathrm{E}\right.$ of $\left.\mathrm{N}\right)$ & 53 & 53 & 53 & 53 & 233 \\
\hline Sea temperature $\left({ }^{\circ} \mathrm{C}\right)$ & 16.8 & 16.2 & 17.2 & 17.4 & 17.4 \\
\hline Cloud cover & Clear $0 / 8$ & Clouds $8 / 8$ & Clear $0 / 8$ & Clouds $8 / 8$ & Clouds $8 / 8$ \\
\hline Sun zenith angle $\left(^{\circ}\right)$ & 40 & 37 & 32 & 52 & 60 \\
\hline Sun azimuth $\left({ }^{\circ}, \mathrm{E}\right.$ of $\left.\mathrm{N}\right)$ & 126 & 222 & 160 & 256 & 270 \\
\hline Short-wave irradiation $\left(\mathrm{W} / \mathrm{m}^{2}\right)$ & 800 & 250 & 930 & 300 & 130 \\
\hline Long-wave irradiation $\left(\mathrm{W} / \mathrm{m}^{2}\right)$ & 360 & 395 & 340 & 370 & 375 \\
\hline Visibility & 8 & 10 & 12 & 15 & 12 \\
\hline
\end{tabular}

The second set of atmospheric data was constructed by matching the MODTRAN sky with the data from the pyranometer and the pyrgeometer. The cloud model was defined in more detail by setting the base height at $1 \mathrm{~km}$ and the thickness at $1 \mathrm{~km}$. A match between observed and modelled global irradiation was obtained by varying the extinction in the clouds. Figure 1 shows the relation between cloud extinction and short-wave and long-wave irradiation. Shortwave irradiation is the sum of solar irradiation and sky radiance. The results shown in the figure were obtained by integrating the irradiation from sun and sky on a horizontal surface, in the wavelength bands of the pyranometer and pyrgeometer (section 2). The results in the figure show that while the long-wave irradiation weakly depends on cloud extinction, short-wave irradiation can be tuned, with values between those corresponding with clear-sky conditions (extinction zero) and heavy clouds (extinction > 1). The weak dependence of the long-wave irradiation on cloud extinction does not prevent a good match between observed and modelled sky radiance, as it is found that the predicted long-wave irradiation was within a few percent of the observed value for all runs. The dominant parameter for the longwave irradiation is the air temperature at sea level. The results shown in Figure 1 correspond to a fully overcast sky.

While the above method works for overcast conditions with sufficiently dense clouds to prevent direct sun from penetrating the clouds, a different method was used for clear-sky conditions (run IP1 and IP6). One of the parameters that affect solar irradiation that could, in principle be used to match observed and modelled values is the visibility. However, at the signature measurement trial this parameter was measured by human observers and the uncertainty is 
relatively large. (Visibility measuring instruments exist, but these also tend to give less reliable results for good visibility conditions that usually prevail during signature measurement trials.) To avoid using parameters in the MODTRAN input that could also affect sky radiance, the solar irradiation was computed with the standard meteorological data and scaled to fit the observed short-wavelength global irradiance. It is noted that this approach could not be taken with the sky irradiation, as in that case both the short-wave and long-wave sky irradiation would be affected.
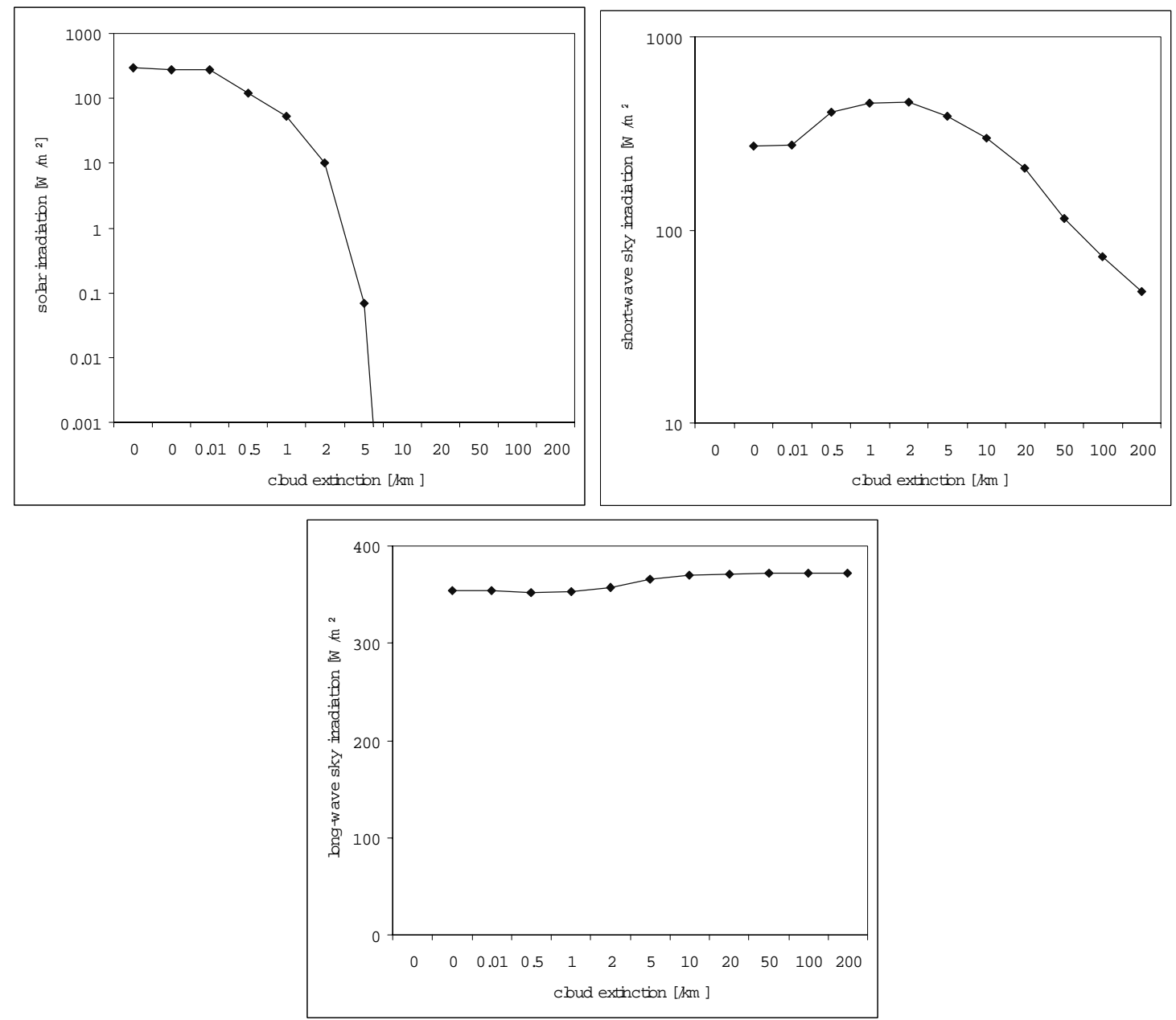

Figure 1. Dependence of short-wave and long-wave irradiation on cloud extinction. The MODTRAN standard cloud model 4 was used (stratus/stratocumulus), with base height $1 \mathrm{~km}$ and thickness $1 \mathrm{~km}$. The meteorological data from run IP7 (see Table 1) were used to describe the atmosphere. Top left: solar irradiation; top right: short-wave sky irradiation; below: longwave sky irradiation. Note that the top two panels use a logarithmic scale. The short-wave and long-wave irradiation was computed by integrating sky radiance and solar irradiance on a horizontal surface, in the wavelength bands of the pyranometer and pyrgeometer used in the trial (see section 2).

Table 2 shows the agreement between short-wave and long-wave irradiation for the five runs. The table also lists the cloud extinction values used in runs under overcast skies (IP4, IP7 and IP8). The scale factors used for the solar irradiation are listed as 'sun amplification factor'. A value larger than 1 indicates that solar irradiation was underpredicted by MODTRAN, using the meteorological parameter listed in Table 1. The results in Table 2 show that after using the cloud extinction coefficient (runs under overcast skies) or scaling the solar irradiation (clear-sky runs) the modelled atmospheric irradiance from sun and sky matches the observed values to within a few percent. In contrast, the modelled irradiance values found without taking pyranometer and pyrgeometer data into account may lead to significant 
errors. Short-wavelength irradiance is underpredicted by up to than $60 \%$ (run IP7), corresponding to an irradiance in the model that is about $190 \mathrm{~W} / \mathrm{m}^{2}$ too low. The total irradiance (short-wave plus long-wave) is underpredicted by up to $30 \%$ (run IP7). These errors are propagated into the signature prediction, where they may be interpreted as shortcomings in the representation of the target or in the signature prediction algorithms.

Table 2: Short-wave and long-wave global irradiation, computed with MODTRAN 4.0, with ('matched') and without ('not matched') the use of pyranometer and pyrgeometer data. Observed values and the difference (in percentages) between the two sets of atmospheric data and observed values are listed. All irradiance values are given in units of W/m², in the wavelength bands $0.33-2 \mu \mathrm{m}$ (short-wave irradiance) and 4.2-40 $\mu \mathrm{m}$ (long-wave irradiance).

\begin{tabular}{|c|c|c|c|c|c|}
\hline & IP1 & IP4 & IP6 & IP7 & IP8 \\
\hline \multicolumn{6}{|l|}{ Observed values } \\
\hline Long-wave sky irradiance & 360 & 395 & 340 & 370 & 375 \\
\hline Short-wave sun and sky irradiance & 800 & 250 & 930 & 300 & 130 \\
\hline \multicolumn{6}{|l|}{ MODTRAN, matched } \\
\hline Long-wave sky irradiance & 361 & 389 & 346 & 370 & 368 \\
\hline Short-wave sky irradiance & 427 & 250 & 403 & 300 & 132 \\
\hline Short-wave sun irradiance & 378 & 0 & 536 & 0 & 0 \\
\hline Cloud extinction $\left(\mathrm{km}^{-1}\right)$ & 0 & 40 & 0 & 10 & 30 \\
\hline Sun amplification factor & 1.25 & 1 & 1.18 & 1 & 1 \\
\hline \multicolumn{6}{|l|}{ MODTRAN, not matched } \\
\hline Long-wave sky irradiance & 361 & 389 & 346 & 369 & 364 \\
\hline Short-wave sky irradiance & 427 & 194 & 403 & 113 & 72 \\
\hline Short-wave sun irradiance & 302 & 0 & 455 & 0 & 0 \\
\hline \multicolumn{6}{|c|}{$\begin{array}{l}\text { Difference observed values and matched sun and } \\
\text { sky radiance }\end{array}$} \\
\hline Difference in long-wave sky irradiance (\%) & 0.3 & -1.6 & 1.8 & 0.1 & -1.8 \\
\hline Difference in short-wave sky irradiance (\%) & 0.6 & 0.0 & 1.0 & -0.1 & 1.5 \\
\hline Difference in total irradiance budget (\%) & 0.51 & -0.98 & 1.19 & 0.01 & -0.97 \\
\hline \multicolumn{6}{|c|}{$\begin{array}{l}\text { Difference observed values and unmatched sun } \\
\text { and sky radiance }\end{array}$} \\
\hline Difference in long-wave sky irradiance (\%) & 0.3 & -1.5 & 1.8 & -0.4 & -2.9 \\
\hline Difference in short-wave sky irradiance (\%) & -8.8 & -22.5 & -7.8 & -62.4 & -44.8 \\
\hline Difference in total irradiance budget (\%) & -6.0 & -9.6 & -5.3 & -28.1 & -13.7 \\
\hline
\end{tabular}


This approach neglects the fact that a pyranometer measures both solar irradiation and the short-wave irradiation from the sky. Changing the intensity of the solar irradiation should also affect the short-wave sky radiance. However, the instruments used at the trial can not separate the two components. If such separate data were available, a more detailed matching of observed and modelled atmospheric radiance could be achieved.

\section{RESULTS}

The comparison between model predictions and observations was done by considering both apparent and physical temperatures of the ship.

\subsection{Apparent temperatures}

Figure 2 shows a comparison between predicted and observed apparent temperatures in the 3-5 $\mu \mathrm{m}$ band. The figure shows the apparent temperature as derived from the IR images and predicted by the model, using the two sets of atmospheric radiance data as explained in the previous section. The difference between the predictions for the two atmospheric radiance data sets is generally of the order of $1-2{ }^{\circ} \mathrm{C}$, with an occasional large value of almost $4{ }^{\circ} \mathrm{C}$ (run IP7, port side). The agreement between model and data is better for the atmospheric radiance data set that was matched to observed irradiance values, although the agreement varies among the runs. The model predictions are within $1-2{ }^{\circ} \mathrm{C}$ of the observed values, which is acceptable, given that the uncertainty in the observed data is of the order of $0.9{ }^{\circ} \mathrm{C}$ for the $3-5 \mu \mathrm{m}$ band.

The results for the port side of the ship show no difference for the two runs that were performed under clear-sky conditions (right-hand-side panel of Figure 2). This is due to the fact that for the shadow side of the ship (port side in these cases) there is no difference between the two sets of atmospheric radiance. For clear-sky conditions, only the solar irradiation was scaled, not the long-wave sky irradiation.
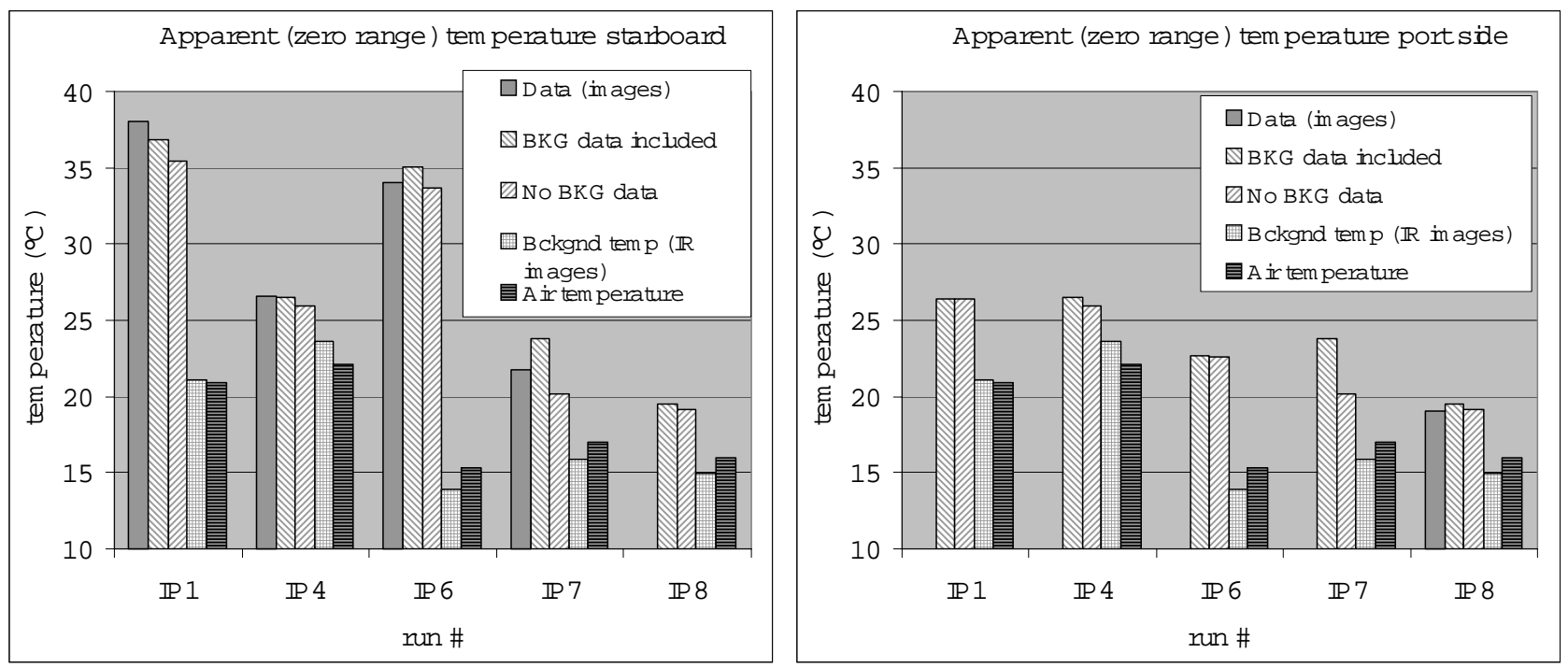

Figure 2. A comparison between observed and predicted apparent temperatures for the starboard side (left) and port side (right). The data series "BKG data included" refers to the results obtained with MODTRAN sun and sky radiance matched to the pyranometer and pyrgeometer data, while the series "No BKG data" refers to the data obtained without considering the irradiation measurements. The apparent temperature were computed with the EOSM model. Data are for the port side of the target were obtained only for run IP8; during the other runs only the starboard side was observed. The uncertainty in the data is $0.9^{\circ} \mathrm{C}$.

The conclusion from the results shown in Figure 2 is that the magnitude of the difference between the model predictions with and without considering the data from the pyranometer and pyrgeometer is of the order of $1-2{ }^{\circ} \mathrm{C}$. This shows that such data must be taken into account, to prevent the mismatch between real and model atmosphere to be interpreted as a 
shortcoming of the signature prediction model or of the ship representation. Unfortunately, the data in Figure 2 do not unequivocally show which of the predicted set of signature data better matches the observed data. In several cases, the observed apparent temperature is in between the two predictions (runs IP6 and IP7), while in other cases the observed value is closer to the prediction for the 'matched' environment (runs IP1, IP4). One factor that affects the influence of global irradiation levels on target signature is the spectral emissivity of the coating of the target. The emissivity was only about $50 \%$ in the short-wavelength band, as a result of which the target temperature is less sensitive to the short-wave global irradiation levels. Nevertheless, the conclusion that must be drawn from the results in Figure 2 is the magnitude of the difference between the signature predictions for the 'matched' and 'non-matched' environments, which is significant.
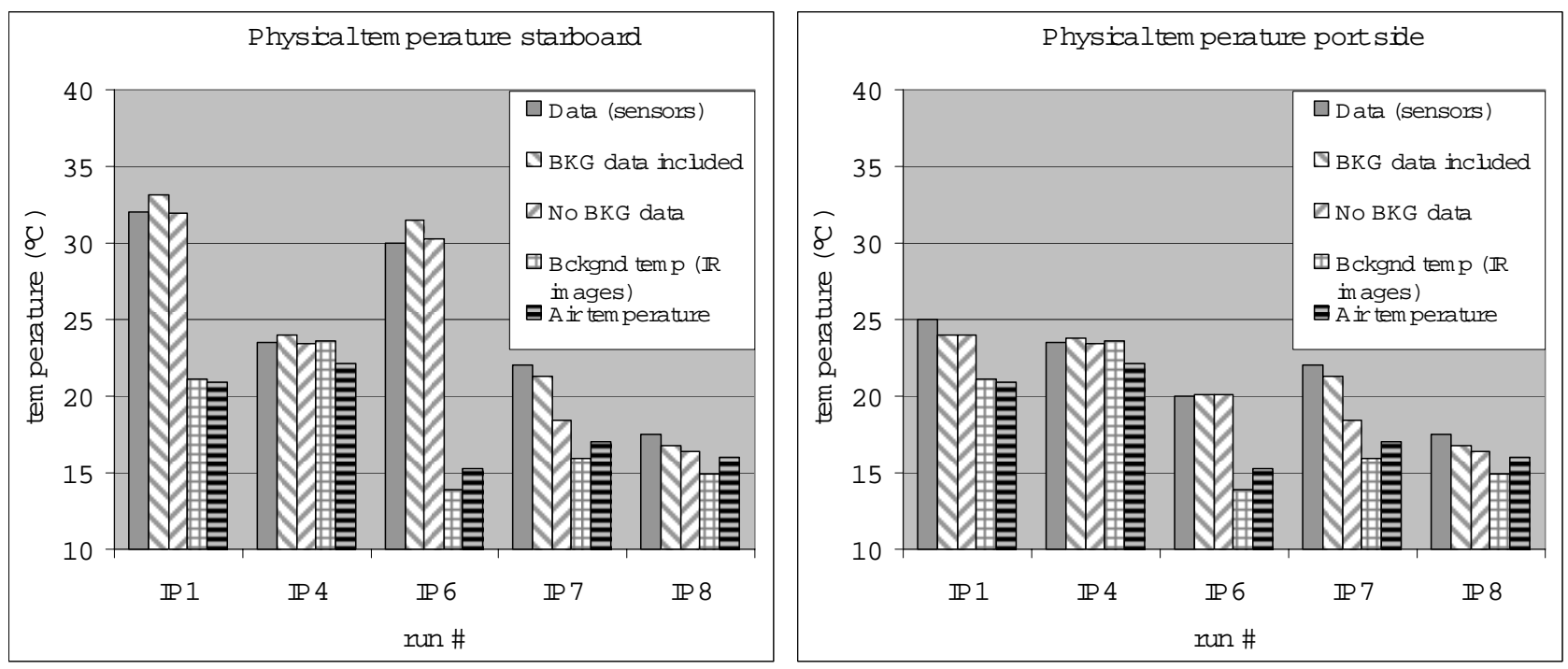

Figure 3. A comparison between observed and predicted physical temperatures for the starboard side (left) and port side (right). The data series "BKG data included" refers to the results obtained with MODTRAN sun and sky radiance matched to the pyranometer and pyrgeometer data, while the series "No BKG data" refers to the data obtained without considering the irradiation measurements. The physical temperature were computed with the EOSM model.

\subsection{Physical temperatures}

Figure 3 shows a comparison between the observed and modelled physical temperatures of the ship. The temperature sensors on the ship provided data on both sides of the ship. The data were taken at the moment the ship passed the measurement site, after a period of about 30 minutes of constant speed and course. During run IP7 the ship had not reached thermal equilibrium and the temperatures for run IP7 are higher than the model predictions. The results show the same trends as observed in Figure 2. The typical difference in model predictions for atmospheric conditions with and without taking irradiation data into account is $1-2{ }^{\circ} \mathrm{C}$.

\section{DISCUSSION}

This paper discusses the validation of infrared signature prediction models, using data measured during a signature measurement campaign. Results are shown to illustrate the use of global irradiation data to minimise the effects of a mismatch between sun irradiation and sky radiance during the trial and in the modelling. The relation between sky radiance and the standard meteorological parameters, such as air temperature, relative humidity, visibility and cloud cover is relatively weak. Using only these parameters does not guarantee that the comparison between signature measurements and model predictions results in a reliable test of the algorithms in the signature prediction model and the representation of the target. Results presented in this paper show that the error in the total irradiance on a single target facet can be as large as $30 \%$. The errors in the apparent or physical temperature of the target computed with a signature 
prediction model depend on the (spectral) emissivity of the coating of the target. The results shown in this paper were derived for a target with a realistic spectral emissivity; temperature changes of up to $4{ }^{\circ} \mathrm{C}$ were found after taking global irradiation data into account. The differences in predicted signatures (i.e., apparent or physical temperature of the target) with and without the use of global irradiation data can be more pronounced for targets with lower spectral emissivity. Low-emissivity coatings are currently being investigated for signature reduction purposes and signature prediction codes must be validated to produce reliable predictions for these new coatings.

As the infrared signature of any target strongly depends on the irradiation of the sun and the sky radiance, the representation of the atmosphere in the modelling must be as close to the trial conditions as possible for a meaningful validation of the signature prediction model. For this, the data from sensors such as a pyranometer and a pyrgeometer are essential. These instruments should be part of the standard equipment used during a signature measurement trial.

\section{ACKNOWLEDGEMENTS}

This work has been done under contracts from the Royal Netherlands Navy.

\section{REFERENCES}

1. Fraedrich, D. and R. Gover, Statistical uncertainty analysis as a tool for model validation, Proc. Third int. symposium on sensitivity analysis of model output, 2001.

2. Fraedrich, D., E. Stark, L.Trygve Heen and C. Miller, ShipIR model validation using NATO SIMVEX experiment results, SPIE Proc. 5075: Targets and backgrounds IX: characterization and representation, 49-59, 2003.

3. Brendhagen, E., and L.Trygve Heen, ShipIR model validation using spectral measurement results from the NATO SIMVEX trial, SPIE Proc. 5075: Targets and backgrounds IX: characterization and representation, 60-71, 2003.

4. Neele, F.P., Infrared ship signature analysis and optimisation, SPIE Proc. 5811: Targets and backgrounds XI: characterization and representation, this volume, 2005.

5. MODTRAN Air Force Research Laboratory web site: http://www.vs.afrl.af.mil/Division/VSBYB/modtran4.html.

6. Kunz, G.K., M.A.C. Degache, M.M. Moerman, A.M.J. van Eijk, F.P. Neele, S.M. Doss-Hammel and D. Tsintikidis, Status and developments in EOSTAR, a model to predict IR sensor performance in the marine environment, SPIE's $11^{\text {th }}$ International Symposium on Remote Sensing, 'Optics in Atmospheric Propagation and Adaptive Systems VII', Maspalomas, Gran Canaria, September 2004. 